\title{
Energy restriction in humans enhances adult hippocampal neurogenesis- associated memory and the longevity protein $\alpha$-klotho
}

\author{
C. Kim ${ }^{1}$, A.M. Pinto ${ }^{2}$, C. Bordoli ${ }^{2}$, L.P. Buckner ${ }^{2}$, P.C. Kaplan ${ }^{2}$, E.J. Jeffcock ${ }^{2}$, \\ I.M. del Arenal ${ }^{2}$, W.L. Hall ${ }^{2}$ and S. Thuret ${ }^{1}$ \\ ${ }^{1}$ Department of Basic and Clinical Neuroscience, Institute of Psychiatry, Psychology \& Neuroscience, King's College \\ London, SE5 $9 R T$ and ${ }^{2}$ Diabetes \& Nutritional Sciences Division, Faculty of Life Sciences and Medicine, King's \\ College London, SE1 9NH.
}

This abstract was presented as the Cellular and Molecular Nutrition Theme highlight.

Adult hippocampal neurogenesis (AHN), the ability to generate new neurons throughout life, occurs in the human hippocampus ${ }^{(1)}$. It has been shown in rodents that AHN is needed for pattern separation, the ability to differentially encode small changes derived from similar inputs, and recognition memory, the ability to recognise previously encountered stimuli ${ }^{(2)}$. Improved spatial learning and recognition memory alongside increased cell proliferation and neuron numbers in the hippocampus is seen in daily calorie restricted mice $^{(3)}$. Increased survival of newly generated neurons in the dentate gyrus and enhanced learning and memory has been reported in intermittent fasting mice ${ }^{(4,5)}$. Evidence that nutrition can significantly affect brain structure and function is increasing substantially. However, previous works have failed to look at the effects of calorie-restricting interventions on AHN-related cognition and this study aims to fill this gap in knowledge. We hypothesised that energy restriction would improve neurogenesis-associated memory and increase $\alpha$-Klotho levels.

Pattern separation and recognition memory was measured in 43 centrally obese (waist circumference $109.1 \mathrm{~cm} \pm 13.4$ ) individuals aged 35-75 years, after a 4-week dietary intervention of continuous energy restriction (CER) or intermittent energy restriction (IER, the "5:2 diet") with an equivalent average weekly energy deficit, using the mnemonic similarity task. Enzyme-linked immunosorbent assay was performed to determine levels of $\alpha$-klotho, an ageing suppressor and life-span extending protein, in the serum as a potential associated-measure of AHN.

Significantly improved pattern separation was observed in both groups, as determined by lure discrimination index score, which indicates pattern separation performance. There was no significant difference in cognitive improvement between diets. IER reduced recognition memory but between-group differences were not statistically significant. Likewise, serum $\alpha$-Klotho concentrations were significantly increased after both dietary interventions.

This is the first human study to investigate the association between energy restriction and neurogenesis-associated cognitive function. It is also the first to explore the effect of a dietary invention on human $\alpha$-klotho levels. Energy restriction may have a beneficial effect on pattern separation and serum klotho levels and could benefit those in an ageing population with declining cognition. Further work is required with a larger sample size, duration and a non-energy restriction control arm.

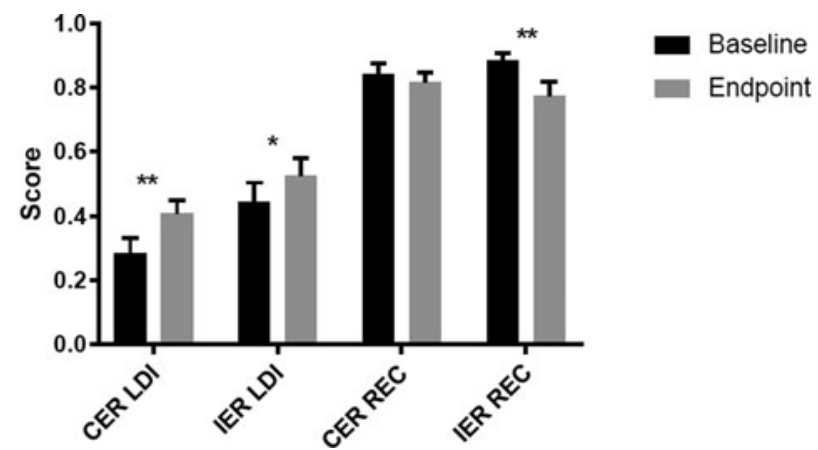

Fig. 1. Both diets improve pattern separation but only IER affects recognition memory. Data presented as mean + SEM. CER:

continuous energy restriction; IER: intermittent energy restriction; LDI: lure discrimination index; REC: recognition. No group differences were observed. $* \mathrm{P}<0.05, * * \mathrm{P}<0.01$ (Paired $\mathrm{T}$ Test between baseline and endpoint).

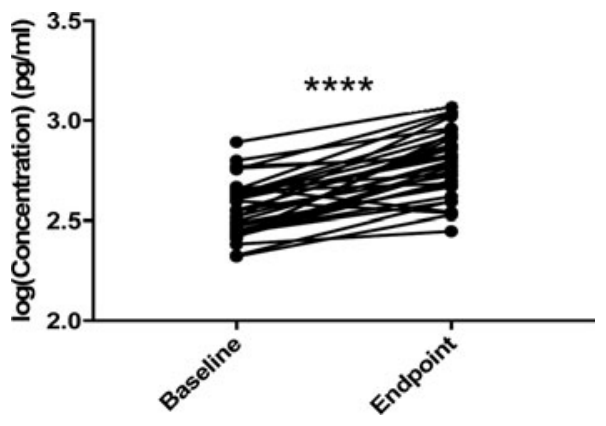

Fig. 2. $\alpha$-Klotho levels are significantly increased following energy restricting dietary interventions. ${ }^{* * * *} \mathrm{P}<0.0001$ (Paired T Test). 